Provided for non-commercial research and education use. Not for reproduction, distribution or commercial use.

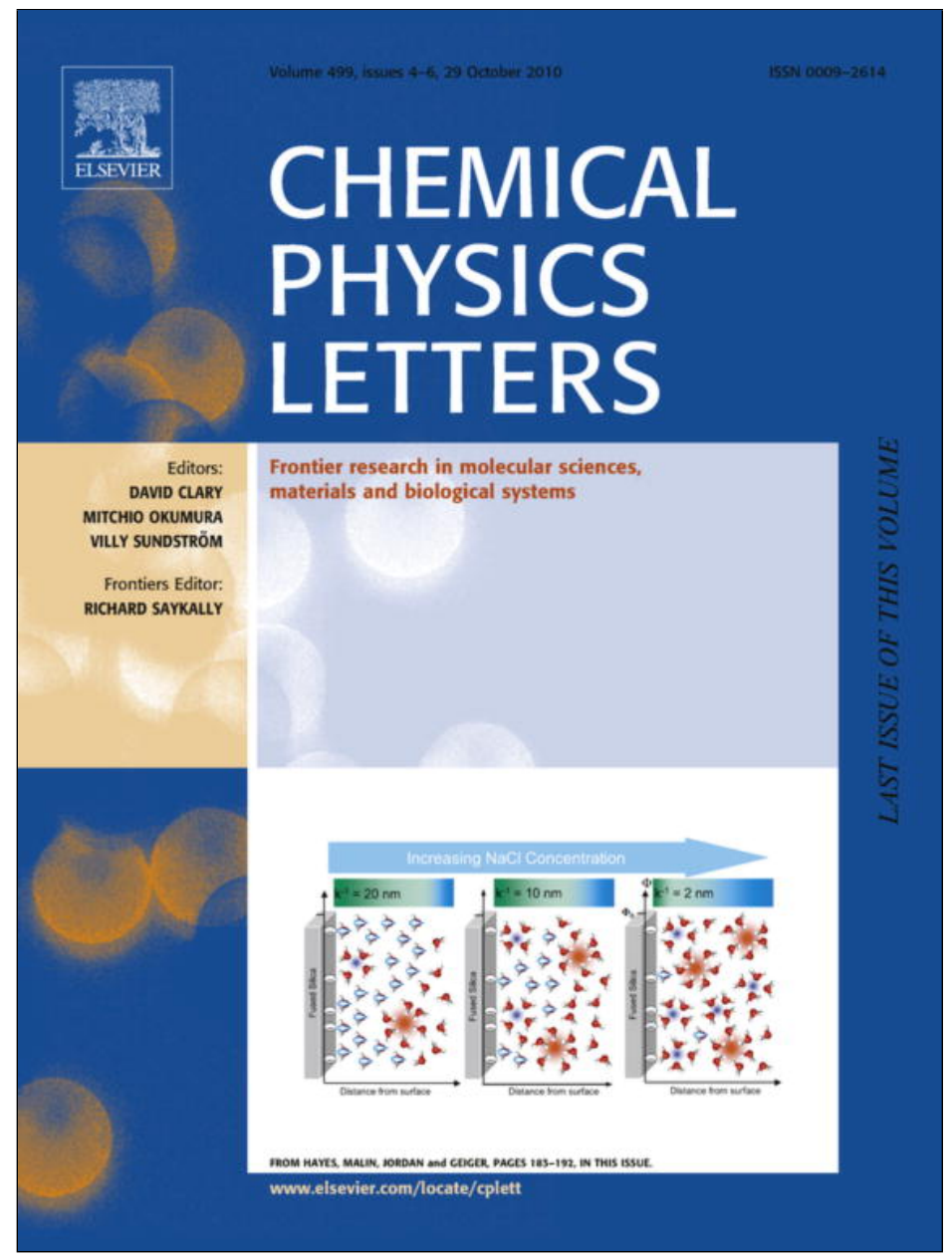

This article appeared in a journal published by Elsevier. The attached copy is furnished to the author for internal non-commercial research and education use, including for instruction at the authors institution and sharing with colleagues.

Other uses, including reproduction and distribution, or selling or licensing copies, or posting to personal, institutional or third party websites are prohibited.

In most cases authors are permitted to post their version of the article (e.g. in Word or Tex form) to their personal website or institutional repository. Authors requiring further information regarding Elsevier's archiving and manuscript policies are encouraged to visit:

http://www.elsevier.com/copyright 


\title{
A virtual screening approach for electronic properties of conjugated organic materials using semi-empirical molecular orbital theory
}

\author{
Huyen T. Nguyen ${ }^{a}$, Thanh N. Truong ${ }^{a, b, *}$ \\ ${ }^{a}$ Institute for Computational Science and Technology, Information Technology Park, Vietnam National University - Ho Chi Minh City, 6 Quarter, \\ Linh Trung Ward, Thu Duc District, Ho Chi Minh City, Viet Nam \\ ${ }^{\mathrm{b}}$ Henry Eyring Center for Theoretical Chemistry, Department of Chemistry, University of Utah, 315 South 1400 East, rm 2020, Salt Lake City, Utah 84112, USA
}

\section{A R T I C L E I N F O}

\section{Article history:}

Received 6 August 2010

In final form 17 September 2010

Available online 22 September 2010

\begin{abstract}
A B S T R A C T
This study presents an approach where semi-empirical molecular orbital theory can be used to screen for desirable optical properties of conjugated organic materials including Organic Light Emitting Diode (OLED) molecules prior to more elaborate computational study or actual experiments. Band-gap, HOMO (for ionization potential), and LUMO (for electron affinity) orbital energies from the PM6 method can be adjusted by shifting vertically to reproduce experimental data or accurate calculated data of one reference molecule in the class, and then the shifting constants can be used to estimate properties for other molecules in the class. The approach was tested with five different subclasses of oligothiophene derivatives which have different end-capped groups. Error analyses indicate that this approach is nearly as accurate as TDDFT but cost significantly less.
\end{abstract}

Published by Elsevier B.V.

\section{Introduction}

Applications of conjugated organic materials such as organic light-emitting diodes (OLEDs) based on electroluminescence property of these organic materials [1] in multimedia electronic industry have just been possible in the last few years. This is due to the fact that OLED has a number of properties superior to the currently dominant liquid-crystal displays (LCDs), such as its low driving voltage, high brightness, high contrast, fast response time, wide viewing angle, and high flexibility, etc. [2,3]. During the past decade, many studies have been carried out to improve the performance and enhance the stability of OLEDs [4-7]. However, since blue light-emitting materials are more rapidly aged than green and red ones, OLED devices have shorter life cycle [8]. Increasing the life cycle of OLED materials will continue to be a challenge.

Experimentally there is a well-known reciprocal rule for conjugated polymers [9-12] in which several properties such as band gaps, absorption wavelengths, ionization potential and electron affinity tend to vary linearly as functions of reciprocal chain lengths. Such relationships are of great importance for functional design of new OLED materials. However, deriving such relationships experimentally would be rather time consuming and expensive. There have been a number of computational studies investigating different properties of OLED materials such as thin

\footnotetext{
* Corresponding author at: Henry Eyring Center for Theoretical Chemistry, Department of Chemistry, University of Utah, 315 South 1400 East, rm 2020, Salt Lake City, Utah 84112, USA.

E-mail address: Thanh.Truong@Utah.edu (T.N. Truong).
}

film thickness distribution [13], charge transport property [14], excitation energies [15], as well as quantitative structure-property relationship (QSPR) for optical limiting of organic compounds [16]. The calculated results were able to assist in the design of new materials with desirable optical properties $[17,18]$. With regarding to predicting optical properties from excitation energies of the OLED materials, time dependent density functional method (TDDFT) has been used broadly because of its reliable results for a wide range of systems [19-23]. Nevertheless, because of the increase of molecular size from monomers to oligomers, it is still impractical to treat large oligomers using TDDFT methodology. Therefore, a more cost-effective strategy to assist experimentalists in functional design of OLED materials would be of great interest and valuable. The use of a semi-empirical MO theory would provide a possibility. The accuracy of the semi-empirical MO methods, however, is always in question. Recently, Puzyn et al. [24] found that the use of general semi-empirical MO PM6 and RM1 methods can provide rather accurate descriptors for QSPR models with accuracy comparable to those from DFT calculations. However, the use of standard semi-empirical MO method such as PM6 for virtual screening of excited state properties of conjugated organic materials so far has not been addressed.

The objective of this study is to develop an approach for using a semi-empirical molecular orbital theory as virtual screening tool for conjugated organic materials. Such virtual screening tool will enable quick estimate of optical properties of oligomers before investing more resources for actual synthetic experiments or elaborated TDDFT calculations. Our hypothesis is that semi-empirical MO would be sufficiently accurate to predict the slopes of linear 


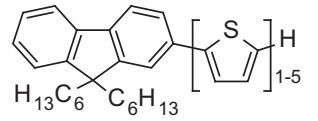

Subclass 1 (1a-e)

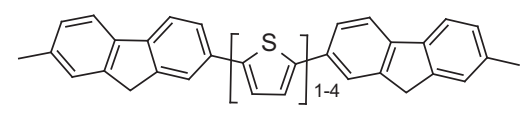

Subclass 2 (2a-d)

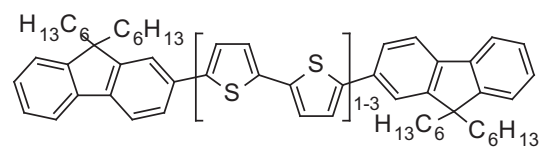

Subclass 3 (3a-c)

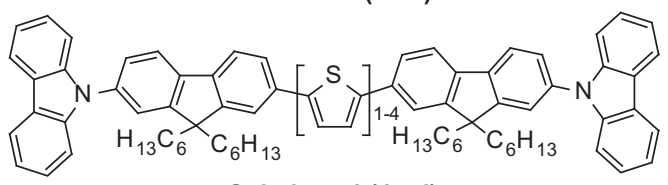

Subclass 4 (4a-d)

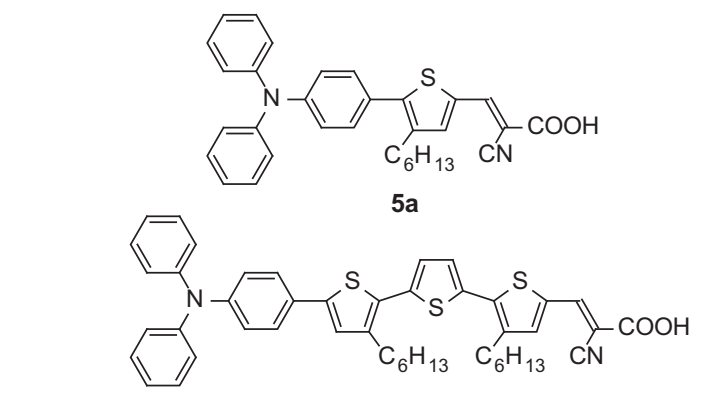

$5 b$

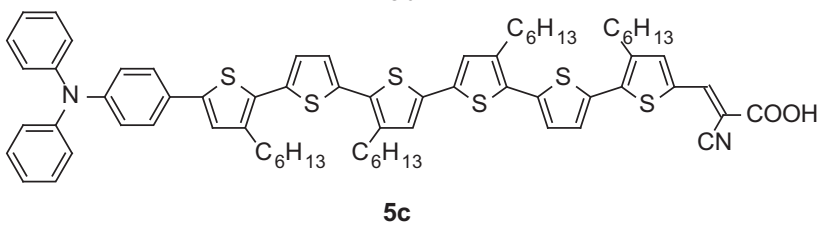

Subclass 5

Figure 1. Molecular structures of subclasses 1-5.

structure-property relationships while the intercepts can be adjusted by TDDFT calculations or experimental data for the monomer or a small oligomer. To test this hypothesis five subclasses 1-5 as shown in Figure 1 all belong to thiophene-based conjugated organic material type [25-29] were chosen since they have sufficient experimental and TDDFT data for comparisons. The chosen subclasses 1-4 have electron donor end-capped groups while sub-class 5 has both electron acceptor and donor end-capped groups. This difference may cause the shift in the optical properties of sub-class 5 differently comparing those of other four subclasses. The electronic properties of the end-capped groups have known to affect the HOMO-LUMO gaps [30]. The use of different end-capped groups enables the range of applicability of the approach proposed here. The results of this error analysis suggest a procedure for how semi-empirical MO method can be used to screen properties of conjugated organic materials.

\section{Methodology}

All calculations were performed using the MOPAC2009 package [31]. The ground-state geometries of the molecules in each subclass were fully optimized with PM6 semi-empirical molecular orbital level of theory without any constraints. The new PM6 method corrects many short-coming in previous well-known AM1 and PM3 semi-empirical MO methods such as having more accurate heats of formation, geometries, hydrogen-bond structures and parameters for many transition metal atoms [32]. The band gap was estimated from the HOMO - LUMO gap from the ground-state orbital energies. Since the proposed methodology is for screening purpose rather being a predictive tool and would be used by mostly bench chemists rather than quantum chemistry experts, more sophisticated methods for calculating excitation energies are not needed since these methods are difficult to employ and thus would discourage the use of the proposed methodology.

\section{Results and discussion}

\subsection{Geometry}

Figure 2 depicts some geometry parameters of molecule $2 \mathrm{~b}$ in ground state including bond lengths, bond angles, and dihedral angles. The calculation results indicate that both PM6 and DFT calculations give non-planar conformation with small differences between bond lengths and bond angles. However, the differences in the dihedral angles fluorene and thiophene rings are larger, namely $28.9^{\circ}$ and $30.1^{\circ}$. These differences lead to the differences in the PM6 band-gap, HOMO, LUMO orbital energies by about $0.15 \mathrm{eV}$. This is within the uncertainty of the shifted PM6 approach as discussed below.

Figure 3 presents the HOMOs and LUMOs of molecules in subclass 1 obtained from PM6 calculation along with the available results from DFT calculations [33]. There is a reasonable agreement between PM6 and DFT results in the characteristics of the HOMO and LUMO orbitals. The HOMOs show anti-bonding character between rings that attributes to the non-planar structures of those molecules at ground state. Meanwhile, the more planar structures at excited states can be explained by the fact that LUMOs show bonding character between rings. PM6 calculations for these molecules with methyl substituents as in previous DFT calculations show that hexyl and methyl substituents yield very little differences on chain planarity.

\subsection{Shifted PM6 method}

There are three important properties of OLED materials that are the band-gap, HOMO and LUMO orbital energies. The band-gap provides information on the color of the light whereas HOMO and LUMO orbital energies are used to estimate the ionization potential and electron affinity of the materials. From our hypothesis, PM6 can be adjusted by shifting vertically to reproduce experimental data (or accurate calculated if experimental data is not available) of one reference point which is often the smallest molecule in the sub-class. This approach is called Shifted PM6 method. The

(a)

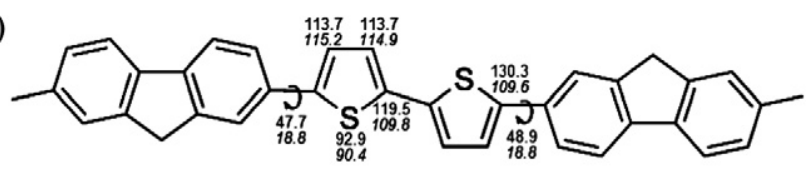

(b)

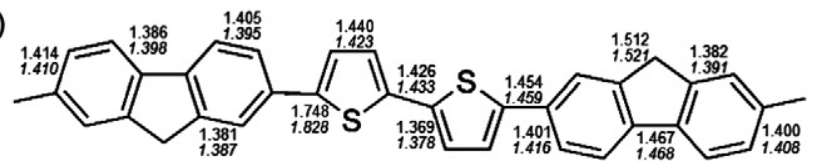

Figure 2. The optimized geometrical parameters of molecules $2 \mathrm{~b}$ using PM6 (normal numbers) and DFT data (italic numbers, taken from Ref. [26]). (a) Angles in degree; (b) Bond lengths in Angstrom. 
HOMO
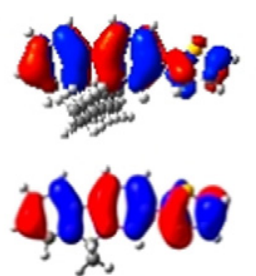

LUMO

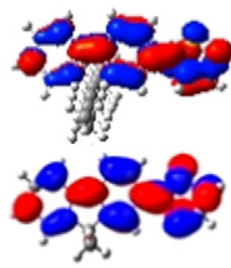

$1 \mathrm{a}$
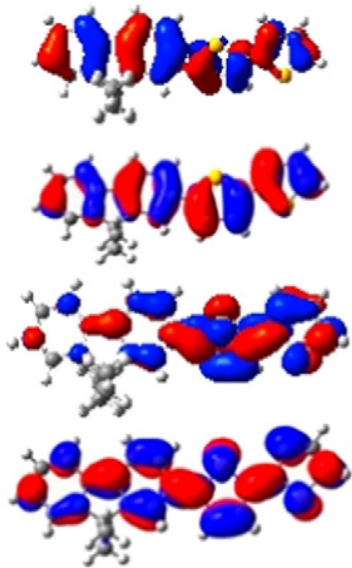

1b

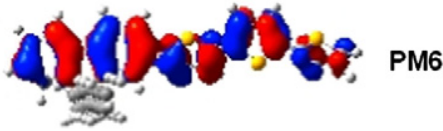

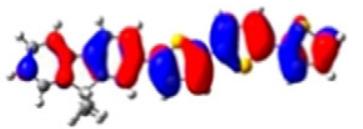

DFT

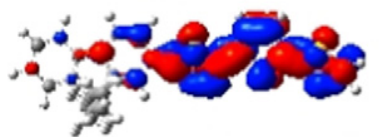

PM6

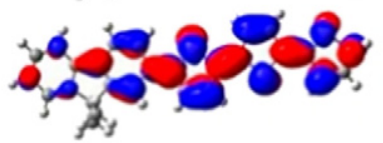

DFT

1c

HOMO
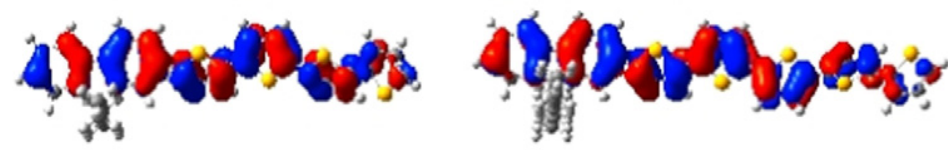

PM6
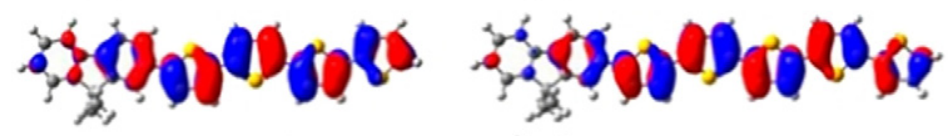

DFT
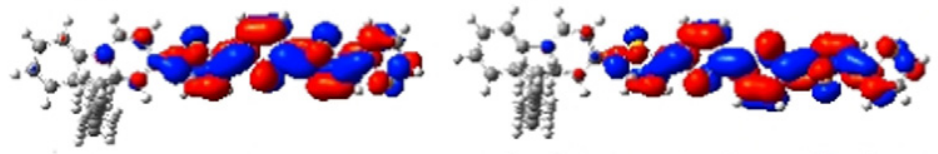

PM6

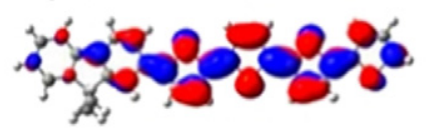

1d

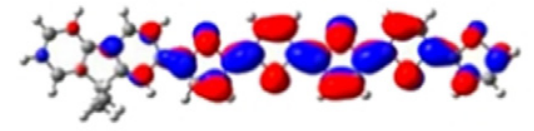

DFT

$1 \mathrm{e}$

Figure 3. HOMO and LUMO of molecules in sub-class 1 using PM6 method and comparison with DFT data (Ref. [33]).

shifting constant can be calculated for each sub-class or averaged over the whole class for a given property. These constants are presented in Table 1. The standard deviation in the averaged shifting constants for all three properties, band gap, HOMO and LUMO energies are relatively small. This suggests that shifting constant determined for a given sub-class can be used for the whole class with acceptable errors of $0.20 \mathrm{eV}$ for the band gap, 0.13 and $0.12 \mathrm{eV}$ for HOMO and LUMO energies.

PM6 band gaps were estimated from the energy difference between the HOMO and LUMO energies. By adding shifting constant for each sub-class, we obtained the shifted PM6 values for molecules in a given sub-class. Alternatively, the PM6 values can be shifted by the average shifting constants for the whole class (see Table 1), i.e. one shifting constant is used for all subclasses. The shifted PM6 band gaps are listed in Table 2 along with TDDFT and experimental data for comparisons. Figure 4 shows that the shifted PM6 band gaps are in good agreement with the experimental ones. Note that for low band gap region there appears to be a consistent overestimation of the shifted PM6 method. Whether this feature is systematic and also exists in other classes

Table 1

Shifting constants $S(\mathrm{eV})$ for the band gap and HOMO energies $(\mathrm{eV})$ for each sub-class and averaged $\mathrm{S}$ for the whole class.

\begin{tabular}{|c|c|c|c|c|c|c|c|}
\hline \multirow[t]{2}{*}{ Sub-class } & \multirow[t]{2}{*}{ Reference molecule } & \multirow[t]{2}{*}{$E_{g}^{\text {Expt. }}$} & \multirow[t]{2}{*}{ HOMO/LUMO } & \multicolumn{2}{|l|}{ PM6 } & \multicolumn{2}{|c|}{$\begin{array}{l}\text { Shifting constant } S \\
(\mathrm{eV})\end{array}$} \\
\hline & & & & $E_{g}$ & HOMO/LUMO & $E_{g}$ & HOMO \\
\hline 1 & $1 \mathrm{a}$ & $3.48^{\mathrm{a}}$ & $-5.65 /-2.17^{\mathrm{a}}$ & 7.96 & $-8.58 /-0.62$ & -4.48 & 2.93 \\
\hline 2 & $2 a$ & $2.92^{\mathrm{b}}$ & $-5.39 /-2.47^{\mathrm{b}}$ & 7.58 & $-8.30 /-0.72$ & -4.66 & 2.91 \\
\hline 3 & $3 a$ & $2.66^{\mathrm{c}}$ & $-5.32 /-2.66^{\mathrm{c}}$ & 7.34 & $-8.41 /-1.07$ & -4.68 & 3.09 \\
\hline 4 & $4 a$ & $2.84^{\mathrm{d}}$ & $-5.34 /-2.59^{d}$ & 7.21 & $-8.12 /-0.91$ & -4.37 & 2.78 \\
\hline 5 & $5 a$ & $2.27^{\mathrm{e}}$ & $-5.47 /-3.20^{f}$ & 6.47 & $-8.23 /-1.76$ & -4.2 & 2.76 \\
\hline Class & & & & & & $-4.48 \pm 0.20$ & $2.89 \pm 0.13$ \\
\hline
\end{tabular}

a Ref. [25].

${ }^{b}$ Ref. [26].

c Ref. [27].

d Ref. [28]; Note: For subclasses $1-4, E_{g}$ was estimated from the onset of absorption spectra $\left(E_{g}=1240 / \lambda_{\text {onset }}\right)$. HOMO was calculated by the empirical equation $\mathrm{HOMO}=-\left(4.44+\mathrm{E}_{\text {onset }}\right)$.

e Ref. [29].

${ }^{\mathrm{f}} \mathrm{E}_{\mathrm{HOMO}}$ was calculated by $\mathrm{E}_{\mathrm{OX}}+4.5 \mathrm{~V}$ (vs. normal hydrogen electrode) $(\mathrm{eV})$ and $\mathrm{E}_{\mathrm{LUMO}}=\mathrm{E}_{\mathrm{HOMO}}-\mathrm{E}_{\mathrm{g}}$. The first oxidation potential $\mathrm{E}_{\mathrm{Ox}}$ values were taken from Ref. [29]. 
Table 2

The shifted PM6 HOMO - LUMO gaps (eV) of five subclasses and comparison with available TDDFT and experimental data. Absolute unsigned errors of shifted values compared with experiment are given in the parentheses.

\begin{tabular}{|c|c|c|c|c|c|c|c|}
\hline \multirow[t]{2}{*}{ Class } & \multirow[t]{2}{*}{ Name } & \multirow[t]{2}{*}{$n_{\text {atom }}$} & \multirow[t]{2}{*}{$n^{\mathrm{a}}$} & \multicolumn{2}{|l|}{$\begin{array}{l}E_{g}^{\text {Shifted PM6 }} \\
(\mathrm{eV})\end{array}$} & \multirow[t]{2}{*}{$\begin{array}{l}E_{g}^{\mathrm{TDDFT}} \\
(\mathrm{eV})\end{array}$} & \multirow[t]{2}{*}{$\begin{array}{l}E_{g}^{\text {Expt }} \\
(\mathrm{eV})\end{array}$} \\
\hline & & & & Each sub-class & Class & & \\
\hline \multirow[t]{5}{*}{1} & $1 \mathrm{a}$ & 66 & 1 & 3.48 & 3.48 & & $3.48^{\mathrm{b}}$ \\
\hline & $1 b$ & 73 & 2 & 3.1 & 3.1 & & 2.99 \\
\hline & $1 \mathrm{c}$ & 80 & 3 & 2.86 & 2.86 & & 2.71 \\
\hline & $1 \mathrm{~d}$ & 87 & 4 & 2.68 & 2.68 & & 2.54 \\
\hline & $1 \mathrm{e}$ & 94 & 5 & 2.65 & 2.65 & & 2.44 \\
\hline \multirow[t]{4}{*}{2} & $2 a$ & 57 & 1 & 2.92 & 3.1 & $3.15^{c}$ & $2.92^{\mathrm{c}}$ \\
\hline & $2 b$ & 64 & 2 & 2.63 & 2.81 & 2.73 & 2.83 \\
\hline & 2c & 71 & 3 & 2.53 & 2.71 & 2.56 & 2.43 \\
\hline & $2 \mathrm{~d}$ & 78 & 4 & 2.4 & 2.58 & 2.37 & 2.26 \\
\hline \multirow[t]{3}{*}{3} & $3 a$ & 130 & 2 & 2.66 & 2.86 & $2.85^{\mathrm{d}}$ & $2.66^{\mathrm{d}}$ \\
\hline & $3 b$ & 144 & 4 & 2.49 & 2.69 & 2.42 & 2.42 \\
\hline & $3 c$ & 158 & 6 & 2.28 & 2.48 & 2.2 & 2.31 \\
\hline \multirow[t]{4}{*}{4} & $4 a$ & 163 & 1 & 2.84 & 2.73 & $2.98^{\mathrm{e}}$ & $2.84^{\mathrm{e}}$ \\
\hline & $4 \mathrm{~b}$ & 170 & 2 & 2.61 & 2.5 & 2.78 & 2.63 \\
\hline & $4 c$ & 177 & 3 & 2.47 & 2.36 & 2.52 & 2.49 \\
\hline & $4 d$ & 184 & 4 & 2.33 & 2.22 & 2.4 & 2.4 \\
\hline \multirow[t]{3}{*}{5} & $5 a$ & 67 & 1 & 2.27 & 1.99 & & $2.27^{\mathrm{f}}$ \\
\hline & $5 b$ & 99 & 3 & 2.08 & 1.8 & & 2.15 \\
\hline & $5 c$ & 156 & 6 & 1.97 & 1.69 & & 2.18 \\
\hline
\end{tabular}

a Number of thiophene units.

${ }^{b}$ Ref. [25].

c Ref. [26].

${ }^{\mathrm{d}}$ Ref. [27].

e Ref. [28]

${ }^{f}$ Ref. [29].

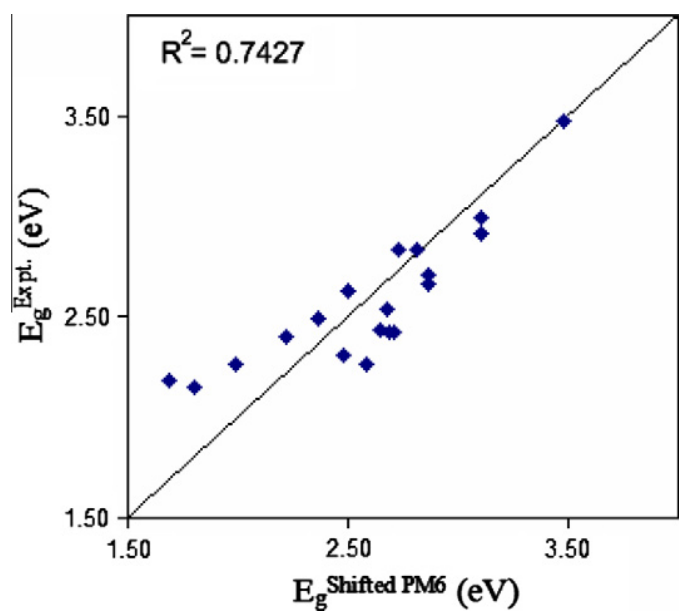

Figure 4. Plot of shifted PM6 band gaps vs. experimental data.

of conjugated organic materials would require further study. A more systematic error analysis, namely the minimum (Min), maximum (Max) and average unsigned errors (AUEs) are presented in Table 3. The difference in AUE for the whole class of the shifted PM6 and available TDDFT band gaps is $0.09 \mathrm{eV}$. It is interesting to note that if using sub-class shifting constants for the band gap and the AUE's and the error ranges of the shifted PM6 method are smaller than those of TDDFT. This confirms our hypothesis that PM6 can accurately predict the trend in a given sub-class. If using the averaged shifting constant for the whole class, the errors of the shifted PM6 method are slightly larger than those of TDDFT.

There are two differences between molecules in sub-class 5 and those in other subclasses, namely the acceptor end-capped group and substituent at thiophene unit. As presented in Table 1, although the shifting constants of sub-class 5 are not significantly different from those of other four subclasses we observe a somewhat
Table 3

Minimums (Min), maximums (Max) and average unsigned errors (AUEs) of calculated band gaps, HOMO and LUMO energies (eV) for each sub-class and class compared with experimental values.

\begin{tabular}{|c|c|c|c|c|c|}
\hline \multirow[t]{2}{*}{ Sub-class } & & \multicolumn{2}{|l|}{$\begin{array}{l}\text { Band gap } \\
(\mathrm{eV})\end{array}$} & \multirow{2}{*}{$\begin{array}{l}\text { HOMO } \\
(\mathrm{eV}) \\
\text { Shifted PM6 }\end{array}$} & \multirow{2}{*}{$\begin{array}{l}\begin{array}{l}\text { LUMO } \\
(\mathrm{eV})\end{array} \\
\text { Shifted PM6 }\end{array}$} \\
\hline & & Shifted PM6 & TDDFT & & \\
\hline \multirow[t]{3}{*}{1} & Min & 0 & & 0 & 0 \\
\hline & Max & 0.21 & & 0.31 & 0.12 \\
\hline & AUE & 0.12 & & 0.17 & 0.05 \\
\hline \multirow[t]{3}{*}{2} & Min & 0 & 0.1 & 0 & 0 \\
\hline & Max & 0.2 & 0.23 & 0.36 & 0.24 \\
\hline & AUE & 0.11 & 0.14 & 0.18 & 0.17 \\
\hline \multirow[t]{3}{*}{3} & Min & 0 & 0 & 0 & 0 \\
\hline & Max & 0.07 & 0.19 & 0.22 & 0.25 \\
\hline & AUE & 0.03 & 0.1 & 0.13 & 0.11 \\
\hline \multirow[t]{3}{*}{4} & Min & 0 & 0 & 0 & 0 \\
\hline & Max & 0.07 & 0.15 & 0.19 & 0.35 \\
\hline & AUE & 0.03 & 0.08 & 0.09 & 0.18 \\
\hline \multirow[t]{4}{*}{5} & Min & 0 & & 0 & 0 \\
\hline & Max & 0.21 & & 0.23 & 0.05 \\
\hline & AUE & 0.09 & & 0.12 & 0.02 \\
\hline & Min & 0 & 0 & 0.01 & 0.01 \\
\hline \multirow[t]{2}{*}{ Class } & Max & 0.49 & 0.23 & 0.42 & 0.27 \\
\hline & AUE & 0.2 & 0.11 & 0.2 & 0.12 \\
\hline
\end{tabular}

large error range in this case. In particular, the largest band gap unsigned error of $0.49 \mathrm{eV}$ belongs to molecule $5 \mathrm{c}$ in sub-class 5 .

Replacing the hexyl group at thiophene unit by hydrogen atom in sub-class 5 changes the band gap, HOMO and LUMO energies by $0.01,0.03$ and $0.02 \mathrm{eV}$, respectively. This further extends our earlier finding that these substituents at thiophene unit have little effect on the electronic properties. We have also tested the effect of end-capped group electronic characteristics by replacing the cyanoarcrylic acid by triphenylamine in sub-class 5 . This results in the differences of $1.02,0.27$, and $1.29 \mathrm{eV}$ for the band gap, HOMO and LUMO energies, respectively. This suggests that the electronic characteristics of end-capped groups have larger effects on the considered properties. This suggests the application of the screening approach here should be used for molecules in the same sub-class with the same end-groups but for different number of oligomer unit.

\section{Conclusion}

In conclusion, the virtual screening approach based on the PM6 semi-empirical MO theory is proven to be a cost-effective method to estimate the optical properties of OLED material providing these properties are shifted by constants to reproduce the experimental or accurate calculated data for one reference point. Error analyses indicate that this approach has nearly the same level of accuracy as of TDDFT but cost significantly less. The screening approach here is for extrapolating TDDFT band gap, HOMO and LUMO orbital energies to larger oligomers where the calculations are computationally prohibitive and for screening classes for desirable properties prior to more accurate calculations and experiments. It is not meant to replace TDDFT where properties including those considered here are systematically more accurate.

\section{Acknowledgment}

The authors thank the Institute for Computational Science and Technology at Ho Chi Minh city, Viet Nam for financial and computer time support.

\section{References}

[1] M. Kope, H.P. Kallmann, P.J. Magnante, Chem. Phys. 38 (1963) 2040.

[2] O. Prache, Displays 22 (2001) 49. 
[3] G.G. Malliaras, R.H. Friend, Phys. Today 58 (2005) 53.

[4] J.D. Froehlich, R. Young, T. Nakamura, Y. Ohmori, S. Li, A. Mochizuki, Chem. Mater. 19 (2007) 4991.

[5] B.H. Wallikewitz, D. Hertel, K. Meerholz, Chem. Mater. 21 (2009) 2912.

[6] F. Villani et al., Phys. Chem. C 113 (2009) 13398.

[7] J. Wu, M. Agrawal, H.A. Becerril, Z. Bao, Z. Liu, Y. Chen, P.A.C.S. Peumans, NANO 4 (2010) 43.

[8] Li, Z.; Meng, H. Ed. Organic Light-Emitting Materials and Devices; CRC Press; Florida, 2006.

[9] M.D. Watson, A. Fechtenkötter, K. Müllen, Chem. Rev. 101 (2001) 1267.

[10] L. Yang, A.-M. Ren, J.-K. Feng, J.-F.J. Wang, Org. Chem. 70 (2005) 3009.

[11] P.F.H. Schwab, J.R. Smith, J. Michl, Chem. Rev. 105 (2005) 1197

[12] J. Bras, S. Guillerez, B. Pépin-Donat, Chem. Mater. 12 (2000) 2372.

[13] E. Lee, Vacuum 83 (2009) 848.

[14] S. Dong, W. Wang, S. Yin, C. Li, J. Lu, Synth. Met. 159 (2009) 385.

[15] S. Grimme, E.I. Izgorodina, Chem. Phys. 305 (2004) 223.

[16] P. Lind, C. Lopes, K. Öberg, B. Eliasson, Chem. Phys. Lett. 387 (2004) 238

[17] Y. Liu, X. Tao, F. Wang, X. Dang, D. Zou, Y. Ren, M. Jiang, Org. Electron. 10 (2009) 1082.

[18] M. Zhang, Z.-S. Li, J.-Z.J. Sun, Mol. Struct. 919 (2009) 204.
[19] J. Tao, S.J. Tretiak, Chem. Theory Comput. 5 (2009) 866.

[20] P.J.J. Hay, Phys. Chem. A 106 (2002) 1634.

[21] G.R. Hutchison, M.A. Ratner, T.J.J. Marks, Phys. Chem. A 106 (2002) 10596

[22] X. Li, X. Liu, Z. Wu, H.J. Zhang, Phys. Chem. A 112 (2008) 11190.

[23] C.R.D. Silva, J. Li, Z. Zheng, L.R.J. Corrales, Phys. Chem. A 112 (2008) 4527.

[24] T. Puzyn, N. Suzuki, M. Haranczyk, J.J. Rak, Chem. Inf. Model. 48 (2008) 1174.

[25] V. Promarak, A. Punkvuang, D. Meunmat, T. Sudyoadsuk, S. Saengsuwan, T. Keawin, Tetrahedron Lett. 48 (2007) 919.

[26] L. Yang, J.-K. Feng, A.-M.J. Ren, Mol. Struct. 758 (2006) 29.

[27] V. Promarak, A. Punkvuang, S. Jungsuttiwong, S. Saengsuwan, T. Sudyoadsuk, T. Keawin, Tetrahedron Lett. 48 (2007) 3661.

[28] V. Promarak, A. Punkvuang, T. Sudyoadsuk, S. Jungsuttiwong, S. Saengsuwan, T. Keawin, K. Sirithip, Tetrahedron 63 (2007) 8881.

[29] P. Shen et al., Dyes Pigments 83 (2009) 187.

[30] J. Nishida, Naraso, S. Murai, E. Fujiwara, H. Tada, M. Tomura, Y. Yamashita, Org. Lett. 6 (2004) 2007.

[31] http://openmopac.net/MOPAC2009.html.

[32] J.J.P.J. Stewart, Mol. Model. 13 (2007) 1173.

[33] S. Jungsuttiwong, S. Namuangrak, V. Promarak, T. Sudyoadsuk, S. J. Vannarat, Theor. Comput. Chem., in preparation. 